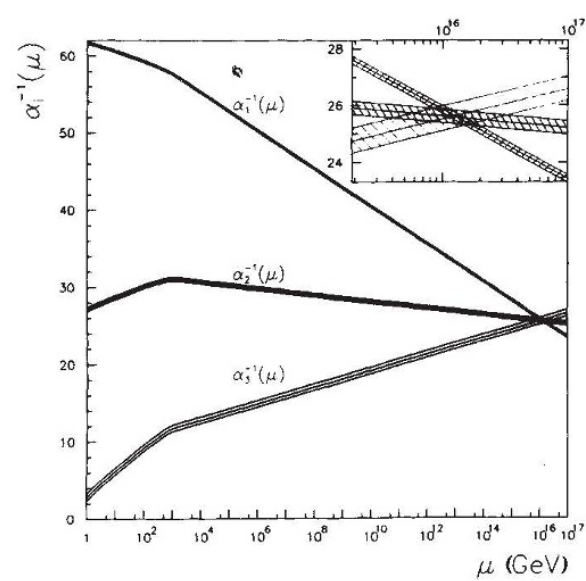

FIG. 2 The evolution of the couplings in the supersymmetric version of the standard model. The fact that the couplings meet at a point is in agreement with simple GUT predic tions such as supersymmetric SU(5) which break in a single step to the standard model; in this case $M_{\mathrm{x}}$ is large enough adequately to inhibit proton decay ${ }^{9}$. The break in the slope of the curves corresponds to the threshold for the new supersymmetric states and agreement with the GUT predictions is maintained only if the masses of the new supersymmetric states lie in the range $10^{2}-10^{4} \mathrm{GeV}$.

become distinct) and the associated $\mathrm{W}^{ \pm}$and $\mathrm{Z}^{0}$ masses up to $M_{\mathrm{X}}$, some thirteen orders of magnitude too large.

This implies that a self-consistent theory of Grand Unification requires an extension of the standard model to include supersymmetry to protect it against such radiative corrections. Moreover to be effective, the symmetry must not be broken at an energy scale much larger than the electroweak breaking scale and hence, as I will discuss next, new supersymmetric states should be relatively light and accessible to experimental detection.

The extension of the standard model to include supersymmetry requires the addition of many new states. These include the 'squarks' and 'sleptons', spin-zero partners of the spin-one-half quarks and leptons needed in the standard model. Also needed are the gluinos, the Wino, the Zino and the photino, spin-one-half partners of the gauge bosons of the standard model, the gluon, the $\mathrm{W}^{ \pm}$, the $\mathrm{Z}^{0}$ and the photon. Although these new states are thought to be considerably heavier than their partners, and hence should not yet have been found directly in laboratory experiments, they will contribute to the radiative corrections. The exciting observation is that their inclusion brings the predictions of grand unification into remarkable agreement with experiment.

The effect of supersymmetry on grandunified predictions has been known for many years $^{6-8}$. Initially the non-supersymmetric SU(5) predictions seemed to be favoured but the recent experimental improvement in the precision measurements of the gauge couplings, largely through the precision measurements of the $\mathrm{Z}^{0}$-boson coupling at LEP, has led to the conclusion that the supersymme- tric prediction is quantitatively better than the non-supersymmetric result. This may be seen in Fig. 2 where, in addition, it may be seen that the precision is now so good that we may even estimate the mass that the new supersymmetric states must have if their radiative effects are to bring the prediction into agreement with experiment. Amaldi $e t$ $a l^{2}$ find that the squarks, sleptons and gauginos should have an average mass in the range $10^{2}-10^{4} \mathrm{GeV}$. Thus one may argue that there is evidence for new forms of (relatively) light supersymmetric matter and an underlying grand unified theory.

The result is so dramatic that it needs to be evaluated with some caution. The most obvious reservation is that it relies on the theoretical extrapolation of the standard model, albeit with the inclusion of supersymmetry, twelve orders of magnitude beyond the energy scale at which it has been tested. No theory has proved to be so robust in the past, so it is understandable if one views this extrapolation with some caution. Even given the framework of grand unification there is considerable uncertainty, for the relative values of the couplings may differ in different unification schemes. Within the (large) class of theories that give the SU(5) predictions, there are corrections coming from virtual states with mass, around or greater than $M_{\mathrm{X}}$ which are not included in the analysis and which can affect the results substantially. Nonetheless it is remarkable that the simplest possible extension of the standard model to include supersymmetry coupled with the simplest assumption about grand unification yield predictions in detailed agreement with experiment and require a very low scale of mass for the new supersymmetric states.

What are the implications of the predicted spectrum of supersymmetric states? If the lower value of the allowed masses is correct, some supersymmetric states should be accessible to the present accelerators, LEP at CERN or Fermilab in Chicago. So far searches have not produced any evidence of such states. The favoured value of $10^{3} \mathrm{GeV}$ would put the supersymmetric states out of the reach of these machines but firmly in the range of future machines, the Superconducting Super Collider in Texas and the proposed Large Hadron Collider (LHC) at CERN. If so, the new accelerators will be able to open a window on the new supersymmetric world and, indirectly, on the ultimate 'theory of everything' that many physicists think will unite the fundamental forces including gravity.

G. G. Ross is in the Department of Physics, University of Oxford, 1 Keble Road, Oxford OX1 3NP, UK.

\footnotetext{
1. Ellis, J., Kelley, S. \& Nanopoulos, D. V. Phys. Lett. B260, 131-137 (1991); B249, 441-448 (1990).

Amaldi, U., deBoer, W. \& Fürstenau, H. Phys. Lett. B260, 447-455 (1991).

3. Langacker. P. \& Luo, M. Univ. Pennysylvania Preprint UPR-0466T (1991).

4. Georgi, H., Quinn, H. R. \& Weinberg, S. Phys. Rev. Lett. 33, 451-454 (1974)

5. Ross, G.G. Grand Unified Theories (Benjamin/Cummings, 1984)

. Dimopoulos, S. \& Georgi, H. Nucl. Phys. B193, 160 (1981). 7. Ibanez, L. E \& Ross, G. G. Phys Lett. B105, 439 (1982).

8. Einhorn. M. \& Jones, D. R. T. Nucl. Phys. B196, 475-488 (1982).

9. Dimopoulos, S., Raby, S. \& Wilczek, F. Phys. Rev. D24, 1681-1683 (1981)
}

\title{
Nobody nose...
}

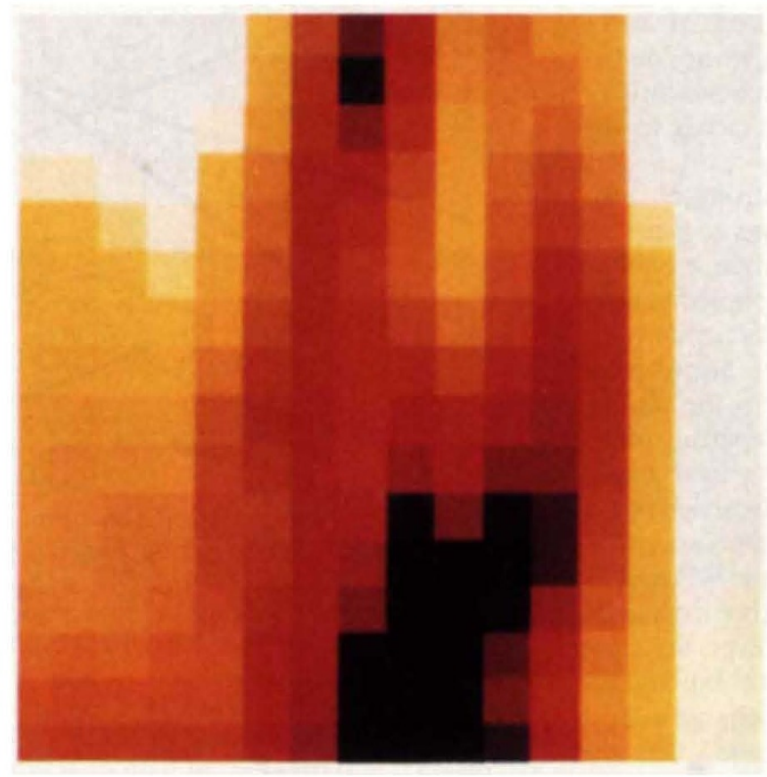

THIS is what ethanol looks like to the simple electronic nose' described by Lundström et al. on page 47. Other gases, such as ammonia and hydrogen, paint a different cubist picture, so that each leaves a distinctive fingerprint. The image is a representation of the variation, caused by the absorption of ethanol, in surface potential of a sensing surface comprising adjacent strips of platinum, iridium and palladium. Top-to-bottom variations, resulting from an imposed temperature gradient, add to the information in the image. The authors make the physiological analogy on the basis of one of the prevailing views on mammalian olfaction: that the

10,000 or so distinguishable odours are identified by a much smaller number of receptors which send signals to specific locations in the brain, producing a type of spatially resolved neuronal fingerprint. In the device of Lundström et al., some decoding of the image is possible in terms of known chemical behaviour: the low affinity of iridium (the central strip) for ethanol, for example, is clearly visible. 